\title{
Distribution and evolution of high redshift galaxies and AGNs from a multi-wavelength survey
}

\author{
K. Sekiguchi ${ }^{1}$, M. Akiyama ${ }^{1}$, H. Furusawa ${ }^{1}$, \\ J. Furusawa ${ }^{1}$, T. Kodama ${ }^{2}$, M. Ouchi ${ }^{3}$, \\ T. Yamada ${ }^{2}$ and SXDS Team ${ }^{4}$ \\ ${ }^{1}$ Subaru Telescope, National Astronomical Observatory of Japan \\ ${ }^{2}$ Division of Optical and Infrared Astronomy, National Astronomical Observatory of Japan \\ ${ }^{3}$ Space Telescope Science Institute \\ ${ }^{4}$ http://www.naoj.org/Science/SubaruProject/SXDS/who.html
}

\begin{abstract}
We report an on-going blank-field multi-wavelength deep and wide survey of the Subaru/XMM-Newton Deep Survey Field (SXDF). The SXDF has been the focus of a wide range of multi-wavelength observing programs spanning the X-ray to the radio. These observations cover a large enough area (the initial optical imaging covers $\left.\sim 1.3 \mathrm{deg}^{2}\right)$ and depth $(B=28.2)$ that they are not affected by large-scale structures (which exist on tens of Mpc scales) and allow us to study the distribution and evolution of high- $z$ galaxies and AGNs, and thus constrain theories for their formation. Our early results include: i) an indication of the primeval Large Scale Structure (LSS) at $\mathrm{z} \sim 5.7$, ii) an indication for the down-sizing of galaxy formation at $\mathrm{z} \sim 1$, iii) identifications of passively evolving systems, and evidence for early formation and the passive evolution of present-day early-type galaxies, and iv) discovery of a large number of optically obscured QSOs. As for the next step forward - we express our hope to use the next generation optical/IR Extremely Large Telescopes (ELTs) to obtain larger and deeper spectroscopy samples of the high- $z$ objects.
\end{abstract}

Keywords. cosmology: large-scale structure, galaxies: distance and redshifts, active, evolution

\section{Introduction}

Observations of the Universe at a range of look-back times will provide powerful constraints on the formation and evolution models of individual objects and of the large-scale structures (LSSs). Investigating the interplay between individual objects and LSS evolution, and the accurate determination of the global properties of various different classes of objects at high redshifts, require a wide-field and deep survey. In this paper, we present one attempt to provide such an observational dataset. Also, we report our initial results from our survey and we mention roles of the next generation optical/IR ELTs on the studies of the LSSs and the formation and evolution of high- $z$ objects.

\section{The Subaru/XMM-Newton Deep Survey Field (SXDF)}

The Subaru/XMM-Newton Deep Survey (Sekiguchi et al. (2004)), a major new multiwavelength blank-field survey, is designed to provide the best view yet of the LSSs in the distant Universe. The properties of the SXDF are given in Table 1. We also, list the dataset existing and/or expected from the multi-wavelength observations from ultradeep radio imaging with the VLA, sub-mm mapping by the Balloon-borne Large Area 
Table 1. Properties of the SXDF.

\begin{tabular}{lcc} 
Equatorial coordinates (J2000.0) & $02^{h} 18^{m} 00^{s}$ & $-05^{\circ} 00^{\prime} 00^{\prime \prime}$ \\
Galactic coordinates & $169^{\circ} 45^{\prime} 32^{\prime \prime}$ & $-59^{\circ} 45^{\prime} 07^{\prime \prime}$ \\
Ecliptic coordinates & $30^{\circ} 28^{\prime} 12^{\prime \prime}$ & $-17^{\circ} 43^{\prime} 22^{\prime \prime}$ \\
Galactic reddening & $E(B-V)<0.04$ \\
Neutral hydrogen column & $N_{\mathrm{H} \mathrm{I}}<2.23 \times 10^{20} \mathrm{~cm}^{-2}$ \\
Cirrus & $I_{100 \mu \mathrm{m}}<1.3 \mathrm{MJy} \mathrm{sr}^{-1}$ \\
Area coverage & $\sim 1.3 \mathrm{deg}^{2}$ \\
Transverse dimension & $\sim 75 \mathrm{Mpc}$ (comoving) at $\mathrm{z} \sim 1$ \\
& $(\sim 145 \mathrm{Mpc}$ at $\mathrm{z} \sim 3)$ \\
Volume coverage & $\sim 3.8^{7} \mathrm{Mpc}$ to $\mathrm{z} \sim 3$ \\
\hline
\end{tabular}

Sub-millimeter Telescope (Devlin (2004)) and SCUBA (Mortier et al. (2005)), deep midinfrared observations from Spitzer Space Telescope (Lonsdale et al. (2003)), deep nearinfrared imaging with the UK Infrared Telescope (Adamson (2001)), and deep X-ray observations with the $X M M$-Newton observatory in Table 2. Figure 1 gives the layout of the X-ray and the optical imaging observations. It is expected that our extensive multiwavelength dataset will provide photometric redshift information accurate to $\delta \mathrm{z} \leqslant 0.1$ over a wide range of redshift, as well as detailed spectral energy distributions for the vast majority of the objects in the field. The Subaru Telescope optical imaging data will be calibrated and the source catalog and calibrated data will be made available to the public via SXDS Web Page (http://www.naoj.org/Science/SubaruProject/SXDS/).

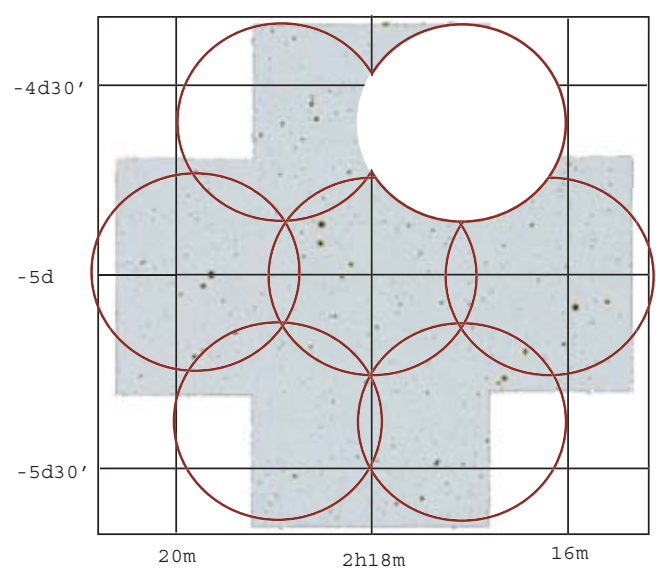

Figure 1. The SXDF optical and X-ray imaging layouts. Five pointings of the Suprime-Cam images were combined to cover $\mathrm{a} \sim 1.3 \mathrm{deg}^{2}$ field centered at R.A. $=02 \mathrm{~h} 18 \mathrm{~m}$, decl. $=-05 \mathrm{~d}$ (J2000). Circles represent 7 pointings of the XMM-Newton observation.

\section{Early results}

The optical imaging with Suprime-Cam on Subaru Telescope and X-ray imaging with the EPIC camera on the ESA XMM-Newton satellite have been completed. The nearinfrared and the sub-mm observations are currently under way. Other wavelength data 
Table 2. A list of major observations of the SXDF

\begin{tabular}{|c|c|}
\hline X-ray by $X M M-N e w t o n$ & $\left(\sim 10^{-14} \mathrm{ergs}_{\left.\mathrm{cm}^{-2} \mathrm{~s}^{-1}\right)}\right.$ \\
\hline \multirow{5}{*}{$\begin{array}{l}\text { Optical imaging } \\
\text { (with Subaru/SuprimeCam) }\end{array}$} & $\left(A B_{\text {mag }}, 3 \sigma, \phi=2^{\prime \prime}\right)$ \\
\hline & $\mathrm{V}=27.5$ \\
\hline & $R=27.6$ \\
\hline & $i^{\prime}=27.5$ \\
\hline & $z^{\prime}=26.5$ \\
\hline \multirow{3}{*}{$\begin{array}{l}\text { Near IR imaging by UKIDSS/UDS } \\
\text { (expected values) }\end{array}$} & $J=25.9$ \\
\hline & $H=25.4$ \\
\hline & $K=24.9$ \\
\hline \multirow{5}{*}{$\begin{array}{l}\text { Near and Mid IR imaging } \\
\left(\text { by SWIRE }{ }^{2}\right)\end{array}$} & $S_{3.6}=7 \mathrm{mJy}$ \\
\hline & $S_{4.5}=10 \mathrm{mJy}$ \\
\hline & $S_{5.8}=27 \mathrm{mJy}$ \\
\hline & $S_{8.0}=32 \mathrm{mJy}$ \\
\hline & $S_{24}=0.4 \mathrm{mJy}$ \\
\hline \multirow{3}{*}{$\begin{array}{l}\text { Submillimeter by } \text { SHADES }^{3} \\
\text { by BLAST } \\
\text { Radio by VLA }\end{array}$} & $S_{850}>8 \mathrm{mJy} \quad\left(\sim 300 \operatorname{arcmin}^{2}\right)$ \\
\hline & 350,450, and $500 \mu m\left(\sim 1 \mathrm{deg}^{2}\right)$ \\
\hline & $1.4 \mathrm{GHz} \quad(\sim 7 \mu \mathrm{Jy})$ \\
\hline
\end{tabular}

are in various stages of reduction and analysis. Here we list some of the early results obtained from the optical and the X-ray data.

Ouchi et al. (2005) obtained a series of narrow-band images of the SXDF to investigate the evolution of the LSSs. In particular, they used the narrow-band N816 filter, with a central wavelength at $\lambda 8150$ and FWHM $=120 \AA$. This corresponds to the redshifted Ly $\alpha$ at $z \simeq 5.7 \pm 0.05$. Combined with the SXDS $R$ - and $i^{\prime}$-band data, they used $\left(i^{\prime}\right.$-NB816) and $\left(R-i^{\prime}\right)$ colours to select 515 LAEs at $z \simeq 5.7$. The density distribution of these LAEs is given in Figure 2. It shows clusters and voids on a $10-40 \mathrm{Mpc}$ scale which are similar to the present-day LSSs. Our follow-up spectroscopy of these clustered LAEs confirmed they are indeed at $z \sim 5.7$. The LAE clusters in the lower south field have spherical shapes, $1 \mathrm{Mpc}$ in diameter (in physical unit, i.e. $\approx 7 \mathrm{Mpc}$ in comoving units). The high star formation rate density (from Ly $\alpha$ fluxes) of these clusters suggests they may be in a burst of galaxy formation phase.

By using the colour slices selection of $1.7<R-z^{\prime}<2.0$ and $0.8<i^{\prime}-z^{\prime}<1.1$, Kodama et al. (2004) constructed a sample of galaxies with $0.9 \lesssim z \lesssim 1$.1. From this sample, they found the large galaxies $\left(\mathrm{M}>8 \times 10^{10} \mathrm{M}_{\odot}\right)$ are predominantly red passively evolving systems while most of the less massive galaxies are blue star forming systems. The age gradient seen in this sample suggests "down-sizing" galaxy formation, i.e. that the massive galaxies form earlier than the less massive one.

Yamada et al. (2005) utilized a large number of old passively evolving galaxies (OPEGs) at $z=1$ to study their spacial distribution and number density. They found $10-30 \%$ variations in the number density over the $0.5 \mathrm{deg}$ scale. Also, they found the number density of OPEGs with $M_{B} \sim M_{*}$ is $\approx 40-50 \%$ of those of the local colour-selected red galaxies and $\approx 60-85 \%$ of the local morphologically-selected galaxies. These results indicate that about a half of the present-day early-type galaxies were formed much earlier than $z=1$.

Optical and radio surveys indicated that the space density of the ultra-luminous QSOs peaks at $z=2$ and declines at higher redshifts (Fan et al. (2001)). Also, Hasinger et al. (2005) reported that the space density of high-luminosity AGNs reaches a peak around $z=2$ while the space density of low-luminosity AGNs peaks at redshifts below $z=1$, confirming previous findings of a luminosity-dependent density evolution. However, there 


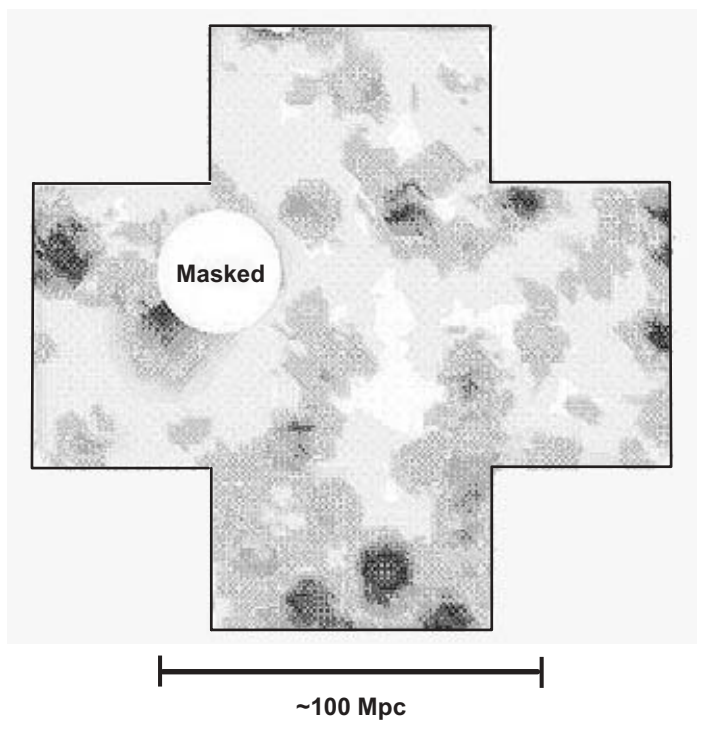

Figure 2. Density Map of LAEs at $z=5.7$ in the SXDF.

are still many faint X-ray sources without optical identifications. Using our deep optical imaging data, we identified the X-ray sources in the SXDF and found a large number of optically-faintX-ray sources. In our X-ray data, these optically-faint sources emerge at flux limit just below $10^{-13} \mathrm{erg} \mathrm{cm}^{-2} \mathrm{~s}^{-1}$ and their fraction peaks $(>30 \%)$ at $10^{-14} \mathrm{erg} \mathrm{cm}^{-2} \mathrm{~S}^{-1}$. These optically-faint X-ray sources contribute $\sim 20 \%$ of Cosmic X-ray Background. Using the X-ray to $R$-band flux-ratio criterion, $\log f_{X} / f_{R}>+1$, we selected 37 of $z \sim 4$ optically obscured QSO candidates. Preliminary spectroscopic observations of 8 of them confirmed all of them are high redshift $(z>3)$ QSOs.

\section{Conclusions}

We presented our initial results from the currently available instruments, which could access the bright end of the populations to $z \sim 3-7$, but for much higher redshifts. For the faint end of the luminosity functions of galaxies and AGNs, even in the moderate redshift range (i.e. $0.7 \lesssim z \lesssim 4.0$ ) requires a larger telescope to obtain the necessary spectroscopy data. In our opinion, the wide and deep field imaging observations are best done with space based instruments. Large aperture ground-based instruments such as the next generation optical/IR ELTs are well suited for faint object spectroscopy. It would be better if the ELTs have wider $(\sim 20-30$ arcsec) FoVs and larger multiplicity (up to a few thousand spectra in one exposure!).

\section{References}

Adamson, A.J. 2001 in: R.G. Clowes, A.J. Adamson, \& G.E. Bromage (eds.), The New Era of Wide Field Astronomy ASP 232, 364

Devlin, M.J. 2004 in: P.H. Rabinovich (ed.), SPIE 5498, 42

Fan, X. et al. 2001, ApJ 121, 54

Hasinger, G., Miyaji, T. \& Schmidt, M. 2005, A\&A 441, 417

Kodama, T. et al. 2004, MNRAS 350, 1005

Lonsdale, C. et al. 2003, PASP 115, 897

Mortier, A.M.J. et al. 2005, MNRAS 363, 563 
Ouchi, M. et al. 2005, ApJ (Letters) 620, L1

Sekiguchi, K.et al. 2004, Astrophisics and Space Science Library 301, 169

Yamada, T. et al. 2005, ApJ 634, 861

\section{Discussion}

Colless: The deep field shows a clear red sequence for the brightest galaxies. Have you used the scatter of the red sequence in the colour-magnitude diagram to constrain the spread in the formation redshifts of these galaxies?

SEKIGUCHI: Yes, that's a thing we need to do, but we haven't got around to do it, yet.

SACKETT: Gorgeous survey. Your early display of the field showed masks that I assume were hiding, that is marking the position of, bright stars. How does the positions of these bright stars compare to the positions of the voids at $z=6$ ?

SEKIGUCHI: Luckily, the bright stars do not overlap with the possible voids at $z=6$.

De Mello: Do you plan on going deeper in the $z$-band? Right now you might have a bias in your higher redshift object because the $z$-band is too shallow to find them.

SEKIGUCHI: Our original goal was about a magnitude deeper than that we obtained so far. In conjunction with the UKIDSS/UDS $J, H, K$ data, we would like to obtain at least a magnitude deeper $z^{\prime}$-band observations with Subaru/Suprime-cam. 